\title{
Exemple d'acquisition et de gestion des écoulements sur le réseau pluvial de la Communauté Urbaine de Bordeaux
}

\author{
P. Briat*, P. Bourgogne** \\ * Lyonnaise des Eaux - Dumez - 91, rue Paulin 33029 Bordeaux Cedex \\ ** Communauté Urbaine de Bordeaux \\ Esplanade Charles de Gaulle - 33076 Bordeaux Cedex
}

Le contexte de l'Assainissement pluvial sur la Communauté Urbaine de Bordeaux sera d'abord décrit : problèmes d'inondation et facteurs de risque; caractéristiques générales du réseau et de ses équipements.

\section{Le contexte de l'assainissement pluvial sur la Communauté Urbaine de Bordeaux (CUB)}

\subsection{Généralités}

La communauté Urbaine de Bordeaux comprend 27 communes. Elle a une superficie de 56000 ha. Elle draine une superficie totale de 90000 ha avec plus de 150 ruisseaux, Esteys et Jalles.

Le réseau d'assainissement a une longueur totale de $2490 \mathrm{~km}$ dont : $901 \mathrm{~km}$ de collecteurs EU, $677 \mathrm{~km}$ de collecteurs EP, $894 \mathrm{~km}$ de collecteurs unitaires (centre de l'agglomération), $18 \mathrm{~km}$ de collecteurs de refoulement.

\subsection{Les problèmes d'inondation et les facteurs de risque}

Le problème majeur de l'assainissement pluvial est celui des risques d'inondation dont les principaux facteurs sont :

La pluviométrie: orages violents en période estivale et parfois fortes hauteurs de précipitations hivernales liées à un climat océanique marqué.

Les conditions aval du milieu récepteur :

La Garonne est le principal exutoire du réseau. Elle est soumise au régime biquotidien des marées avec une amplitude moyenne de $4,5 \mathrm{~m}$ et maximale de $6,8 \mathrm{~m}$ pouvant gêner considérablement l'évacuation gravitaire.

La topographie et l'hydrographie naturelle

13500 ha de part et d'autre de la Garonne sont à une côte inférieure aux plus hautes eaux du fleuve.

D'autre part, le bassin versant a une forme de cuvette et il est drainé par des ruisseaux qui convergent presque tous vers cette partie centrale souvent la plus urbanisée.
L'imperméabilisation croissante des bassins versants

Les réseaux qui n'ont pas été conçu initialement pour une urbanisation aussi développée.

\subsection{Caractéristiques générales du réseau et des équipements}

Collecteurs : La plupart des collecteurs primaires résultent de la canalisation de ruisseaux naturels.

\section{Pompages}

Lorsque le niveau de la Garonne ne permet plus l'évacuation gravitaire des eaux pluviales, celles-ci sont pompées par des stations situées dans les zones basses: 32 stations de pompage EP, débit gobal de pompage de $100 \mathrm{~m}^{3} / \mathrm{s}$ environ.

\section{Bassins de rétention}

Les collecteurs aval sont saturés par l'accroissement de l'urbanisation périphérique. Le grossissement de ces collecteurs par zone très urbanisée posant des problèmes techniques et financiers particulièrement difficiles à résoudre, il a été fait appel à la technique des bassins de retenues implantés sur le cours des grands ruisseaux de façon à réguler et étaler leurs débits.

Actuellement il y a 23 bassins de rétention de divers types (en eau ouvert, à sec ouvert, entérrés) pour une capacité totale de retenue de $1100000 \mathrm{~m}^{3}$ environ.

\section{Métrologie}

Afin de connaître l'état de charge de réseau et la pluviométrie, les équipements de mesure suivants sont installés : 40 pluviomètres dont 16 télétransmis, 40 limnimètres télétransmis, 6 marégraphes télétransmis.

Poste central de télécontrôle

Tout ceci conduit à un ensemble d'installations de plus en plus complexes, très dispersées géographiquement, pouvant interférer entre elles.

Leur gestion est télécontrôlée et télégérée par un poste central situé à la station d'épuration du cours Louis Fargue. Un opérateur est en permanence au poste central de Télécontrôle (Service $3 \times 8$ ). 


\subsection{La gestion du réseau pluvial}

La Communauté Urbaine de Bordeaux a confié la gestion du réseau pluvial à la Lyonnaise des Eaux-Dumez qui intervient ici au niveau de deux départements (Réseaux et Usines).

Le service "Gestion des écoulements" a une place centrale pour cette gestion du télécontrôle et ses fonctions en période normale d'alerte et de crise seront décrites ci-après.

D'autres services assurent également des fonctions importantes dans cette gestion du réseau pluvial (maintenance, études, automatismes, statistiques).

\section{Les besoins du gestionnaire et le rôle du télécontrôle}

\subsection{Historique}

Dès 1966 le besoin s'est fait sentir de pouvoir surveiller à distance et de façon permanente, les stations de pompage par acquisition, télétransmision et regroupement de la signalisation des défauts.

Vers les années 1976 on s'est doté de moyens de télécommande de vannes de bassins de rétention améliorant ainsi considérablement leur efficacité. On a installé des limnimètres télétransmis permettant de mieux connaître le fonctionnement du réseau et de déterminer des "points sensibles ". Pour ce faire a été mis en place le premier vrai système de gestion technique centralisée sous la forme d'un poste 16256 FLUTEC et d'un LSIII/02 de DEC.

Ce système était déjà performant mais de nombreux besoins n'étaient encore pas satisfaits :

- mise en alerte météoroloqique et vision globale de la situation pluviométrique sur la CUB et de son évolution ; - vision rapide de l'état des équipements telle qu'on peut l'avoir par les vues synoptiques et courbes animées ; - enregistrement (numérique) et mise à disposition rapide et facile des divers paramètres acquis par le poste central (mesures, états...);

- amélioration de la sécurité de fonctionnement ;

- gestion optimale des bassins de rétention de façon à utiliser au mieux les équipements existants.

Pour la mise en alerte météorologique, nous nous sommes dotés en 1983 d'un terminal météotel permettant une prévision qualitative des déplacements pluvieux en dehors de la CUB. Sur la CUB il y avait de gros problèmes d'échos de sols qui n'ont été résolus que très récemment (avril 1991).

Pour le reste, dès 1981, débutèrent les études préalables qui ont abouti à la mise récente d'un nouveau système de télécontrôle.

\subsection{Les différentes phases de gestion}

Une des grandes caractéristiques de la gestion de l'assainissement pluvial est d'être soumis aux événements aléa- toires météorologiques qui nécessitent parfois des dispositions particulières de gestion avant, pendant ou après leur occurrence.

C'est pourquoi nous analyserons ici plus précisément les besoins pour les différentes phases de gestion.

\subsubsection{Phase normale}

Elle correspond au temps sec ou aux petites pluies qui ne présentent pas de risques sur le réseau (débordements...). Les besoins associés à cette phase sont :

Surveillance météorologique

Elle est permanente ; il faut en effet pouvoir prévoir à l'avance ( $1 \mathrm{~h}$ environ) le début de pluie sur la CUB est estimer la probabilité de dépassement de seuils pluviométriques critiques présentant un risque de débordement. Ceci est assuré par des relations avec la Météo et une console Météotel.

\section{Surveillance des installations}

Il faut en permanence connaître l'état de fonctionnement des équipements et même parfois procéder aux essais à distance (groupes électrogènes, vannes...). Tous les défauts doivent pouvoir être analysés. L'enregistrement numérique au poste central des paramètres souhaités (télésignalisations, télé-alarmes, télémesures...) est donc une nécessité. La vision globale apportée par les courbes et vues synoptiques animées est également indispensable.

La constitution et l'exploitation de banque de données hydrologiques

(pluviométrie et limnimétrie).

L'objectif est de faire de l'acquisition et du stockage numérique des données pluviométriques et limnimétriques permettant de réaliser les traitements pour les divers besoins temps réel et temps différé :

- pour le temps réel accès rapide aux mesures récentes avec diverses formes de présentation plus ou moins synthétiques ;

- pour le temps différé s'y ajoutent des possibilités diverses de statistiques.

Ces banques de données doivent satisfaire certains critères de qualité qui n'atteignent pas les anciens systèmes de traitement de la mesure :

- permanence des données acquises (pas de trous) qui se réalise par une certaine redondance au niveau du matériel local d'acquisition ;

— sécurité contre les coupures de lignes de télétransmission ;

- synchronisation de tous les appareils d'acquisition locaux y compris ceux de secours;

— horodatage des mesures à la source ;

- mise à disposition d'outils de critique-validation pour sélectionner ou éliminer des mesures, les commenter ou leur affecter un code qualité ;

- élimination de multiples intervenants par une intégration poussée de la chaîne mesure, acquisition transmission, stockage provisoire, critique-validation, stockages définitifs, traitements.

La constitution d'une banque de données hydrologiques de qualité n'est pas un travail à court terme, mais elle est 
très utile aux diverses études liées à la construction ou l'exploitation des réseaux d'assainissement.

\subsubsection{Phase de mise en alerte}

La mise en alerte est destinée à pouvoir mobiliser à temps le personnel et prendre des mesures de sécurité sur le fonctionnement hydraulique du réseau.

L'alerte est déclenchée sur prévision d'une situation météorologique à risque pouvant être le dépassement d'un seuil pluviométrique ou un orage sur le territoire de la C.U.B. Le délai de prévision souhaitée est de 1 à 2 heures.

Cette mise en alerte est généralement effectuée par la Météo Régionale, mais aussi parfois par l'opérateur du télécontrôle lui-même.

Lorsqu'on est en phase d'alerte, l'opérateur du télécontrôle ferme (par télécommandes) la vidange de tous les bassins de rétention de façon à soulager au maximum les collecteurs situés à leur aval et réduire ainsi leurs risques de débordement.

La qualité de la prévision associée à cette mise en alerte est importante car il faut au maximum :

- éviter les fausses alertes car on pollue inutilement certains bassins par les eaux de temps sec;

- éviter la non mise en alerte qui pourrait être bien plus préjudiciable, mais qui heureusement est rare. Elle a surtout lieu lorsque des orages locaux se développent sur le territoire de la C.U.B.

La qualité actuelle de la prévision est assez satisfaisante mais des améliorations sont souhaitables. Ces améliorations pourraient venir d'outils intégrant au mieux les données radar et les données pluviométriques locales de façon à affiner la prévision actuelle et, sur le territoire de la C.U.B., à fournir des images de répartition de pluie plus fines que celles dont on dispose actuellement.

\subsubsection{Phase de crise}

La phase de crise correspond aux périodes de précipitations pluviométriques suffisamment fortes pour solliciter de façon importante les équipements (stations de pompage, bassins...) et le réseau, avec éventuellement des inondations locales ou plus généralisées.

Pendant cette phase, où l'opérateur du télécontrôle se trouve assisté (cadres, maîtrise...) plusieurs fonctions doivent être assurées : Evaluation de la situation globale et de son évolution (météorologie, charge des réseaux...), surveillance du fonctionnement des équipements électromécaniques des stations de pompage et bassins et éventuellement déclenchement d'interventions locales d'urgence, réponse aux multiples appels téléphoniques, gestion des moyens d'intervention sur le terrain (hydrocureuses...).

Dans quelques cas gestion en temps réel des écoulements de façon à minimiser les risques d'inondation.

Pour remplir ces diverses fonctions le systèmes informatique doit offrir de façon ergonomique les aides suivantes : a) Vision globale des paramètres hydrologiques :

- vues synoptiques et courbes animées pour l'état de charge du réseau;

- images de la répartition spatiale de la pluie. Ceci se fait actuellement (isohyètes) toutes les $5 \mathrm{~mm}$ à partir des pluviomètres télétransmis (16), mais pourrait être amélioré par une meilleure intégration des données radar météorologique. Les hyétogrammes viennent compléter ces images.

b) Surveillance du fonctionnement des équipements :

— vision des paramètres (télé-alarmes, télé-signalisation, télémesures) selon les systèmes classiques de G.T.C. ;

- aide au diagnostic des alarmes. (système expert en cours d'élaboration).

\subsubsection{Phase de Post-crise}

La phase de post-crise correspond à la fin prévisible de situation pluviométrique à risque.

Il faut alors vidanger rapidement les bassins de rétention, soit de façon traditionnel (télécommandes) soit à l'aide de régulations dynamiques.

Par ailleurs l'exploitant doit procéder à l'analyse de la crise passée (pluies, fonctionnements, interventions, inondations...). La richesse et la facilité d'exploitation des données du télécontrôle est alors un élément appréciable.

\section{Mise en oeuvre}

L'organisation générale du télécontrôle est décrite sur la figure 1 .

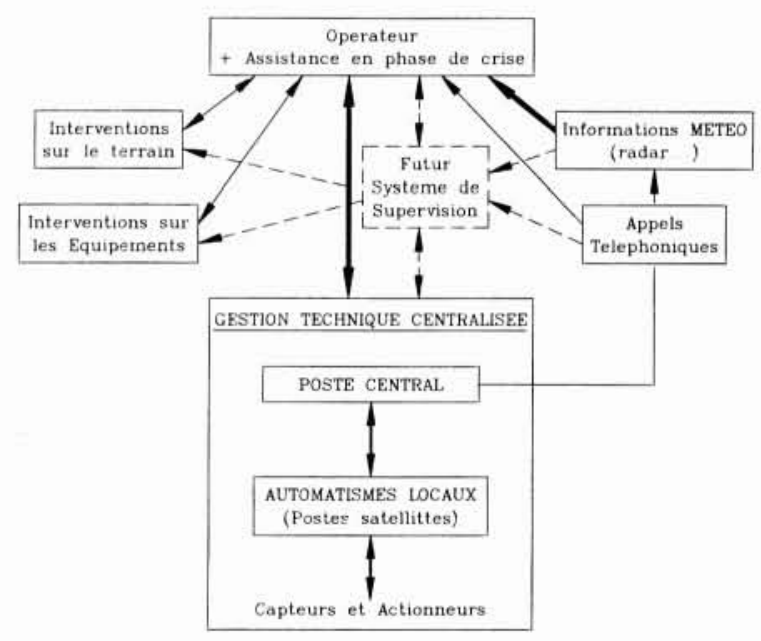

1.

Systemes au Stade dEtudes Prelinnnaires 


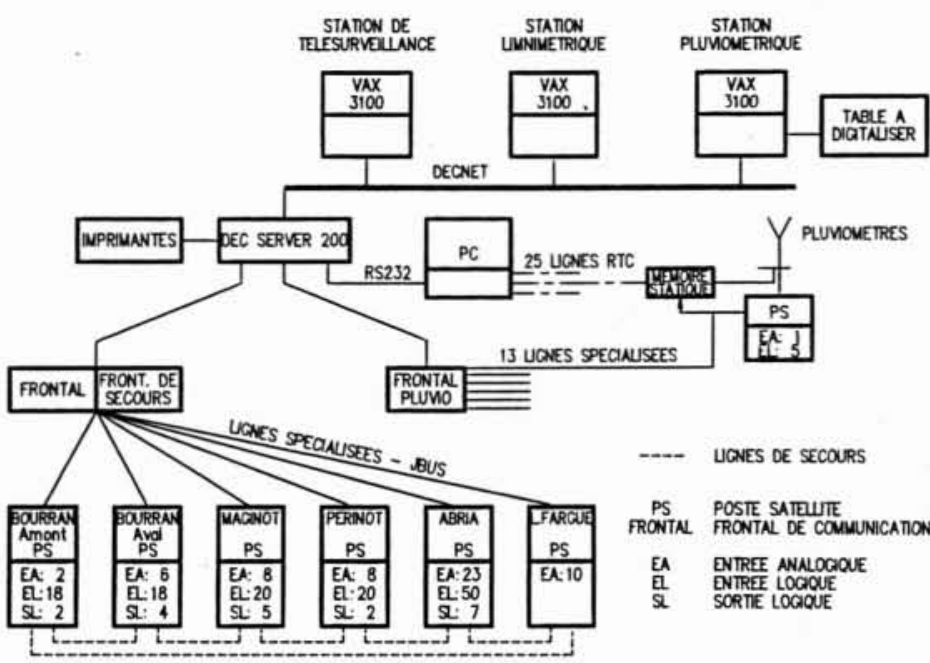

2.

Nous limiterons ici l'exposé à quelques caractéristiques du système de gestion Technique Centralisée qui vient d'être renouvelé (une partie des postes satellites restant cependant encore à raccorder au nouveau Poste Central).

\subsection{Architecture générale de la G.T.C.}

Le nouveau système de G.T.C. actuellement en place est décrit sur la figure 2 .

\subsection{Postes satellites et frontaux}

Les postes satellites sont constitués par des automates programmables.

Les postes frontaux sont des microordinateurs industriels IBM 7532. Un frontal spécifique est réservé aux pluviomètres isolés ( 13 pour le moment).

Une caractéristique intéressante des postes satellites est à noter : il s'agit de l'acquisition structurée en Evénement. Toutes les informations acquises par les postes satellites sont structurées en Evénements stockés en file dans une table spécifique. Pour les mesures les Evénements peuvent être acquis cycliquement ou sur seuil de variation de la mesure.

Les horloges des postes satellites sont synchronisées une fois par jour à partir du Poste Central assurant ainsi une bonne cohérence des horodatages des Evénements.

Le contenu intégral des Tables d'Evénements est déchargé cycliquement (toutes les 3 secondes environ) par le frontal.

Cette structure par Evénements offre de nombreux avantages dont :

- pour les mesures, souplesse du mode d'acquisition (cyclique, par seuil...);

- indépendance de l'horodatage de tous les délais ultérieurs de télétransmission et possibilité de bonne synchronisation ;
- sécurité en cas de coupure de lignes : en effet les Evénements continuent dans ce cas à être stockés dans les Tables et peuvent être récupérés dès rétablissement de la communication.

Comme indiqué sur la figure 2 et le $\S 2$, les pluviomètres sont également munis d'enregistreurs locaux à mémoire statique (CR2M SAB600); l'horloge des enregistreurs est synchronisée une fois par jour par le frontal. Les enregistreurs peuvent être déchargés par un microordinateur à travers le réseau P.T.T. Les données du micro sont ensuite transférées sur le Poste Central.

\subsection{Poste Central}

\subsubsection{Structure générale}

Le système informatique du Poste Central est basé sur 3 stations VS 3100 de DEC connectées en réseau DECNET doté d'un serveur de communication.

Les bases de données INFORMIX sont disponibles sur chaque station.

- la VS 3100 de télésurveillance est pilote de l'ensemble ; elle assure l'acquisition, le stockage dans les banques de données provisoires de télésurveillance, limni et pluvio, la télésurveillance et enfin la régulation dynamique du bassin de rétention de Bourran;

- la VS 3100 limnimétrie assure les fonctions plus spécialisées de télésurveillance limnimétrique (vue globales...), la constitution et le traitement d'une Banque de Données limnimétrique provisoire et critiquée;

- la VS 3100 pluviométrie effectue les traitements pluviométriques en temps réel (isohyètes, hyétogrammes) et en temps différé (digitalisation, critique de la banque de données pluviométrie, traitements sur la base de données.

Le détail des diverses fonctions de cette station ne sera pas présenté ici, sauf ce qui concerne la régulation de Bourran qui est un exemple de gestion dynamique des écoulements en temps réel.

\subsubsection{La régulation du Bassin de Bourran}

a) Description du réseau

Une régulation automatique du bassin de Bourran $\left(135000 \mathrm{~m}^{3}\right)$ a été mise en œuvre à titre expérimental. Le réseau à l'aval de Bourran est schématisé figure 3 .

Le but de cette régulation est de minimiser le remplissage du bassin pour éviter son débordement.

b) Principes généraux de régulation

Le principe général consiste dans l'utilisation en permanence de la débitance aval tout en évitant les débordements.

Deux types de régulation sont combinés :

La régulation en boucle fermée (feed-back)

Elle consiste à mesurer les hauteurs d'eau du collecteur 


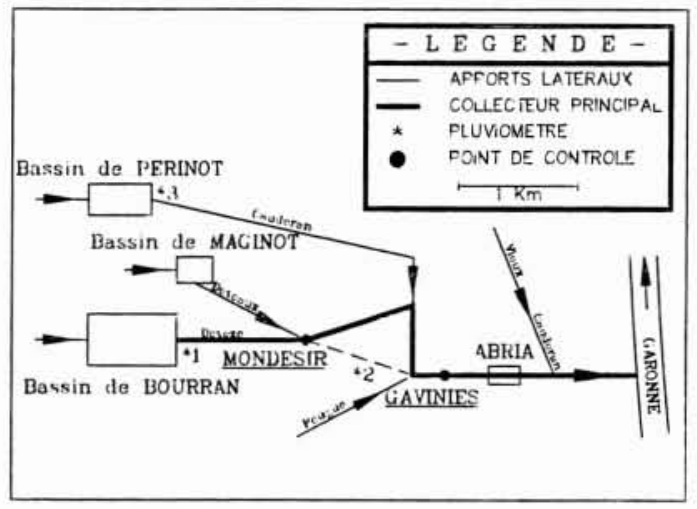

3.

principal aval et à maintenir la hauteur effective aussi proche que possible des hauteurs de consigne fixées par l'opérateur (proche d'un PIO classique).

L'avantage de cette régulation est de compenser automatiquement les perturbations dues aux apports latéraux ; mais elle est un peu lente pour faire face aux variations rapides de ces perturbations.

La régulation en boucle ouverte (feedforward)

Le débit de sortie de la retenue doit être adapté aux apports latéraux de la Deveze. Pour cela une prévision de ces débits est nécessaire à l'aide d'un modèle hydrologique. Cependant, il n'est pas nécessaire d'avoir des prévisions de pluie puisque le temps de réponse des bassins versants est égal ou supérieur au temps de propagation entre le bassin et les points de contrôle aval.

La régulation boucle ouverte permet d'anticiper les variations d'apports latéraux. Mais cette méthode basée sur des calculs n'a pas de possibilités d'auto-corrections. Elle ne peut donc être utilisée toute seule.

\section{La régulation mixte}

Elle combine les modes de régulation précédents en combinant leurs avantages respectifs. Le schéma général est présenté sur la figure 4 .

c) Etudes préalables à la mise en œuvre

Des études relativement poussées ont été nécessaires pour établir cette régulation : hydrologique, hydraulique, automatique, sécurité... L'étude du fonctionnement par simulation numérique a été ici importante.

La figure 5 montre les résultats d'une simulation pour la pluie du 11 décembre 1981. Le pas de temps de régulation est de $5 \mathrm{~mm}$. La figure montre que la régulation en boucle fermée est plus douce que la régulation mixte, mais aussi moins performante.

On voit ici qu'il est possible de maintenir avec une bonne précision le niveau de Gavinies proche du niveau de consigne fixé, malgré des apports latéraux importants et rapidement variables.

\section{d) Essais et mise en service}

Actuellement cette régulation est réalisée et est en phase finale d'essais. Pour ces essais il faut des pluies qui ne sont que passagères : c'est aussi une difficulté pour la mise au point des systèmes complexes de gestion dynamique des écoulements en assainissement pluvial urbain.

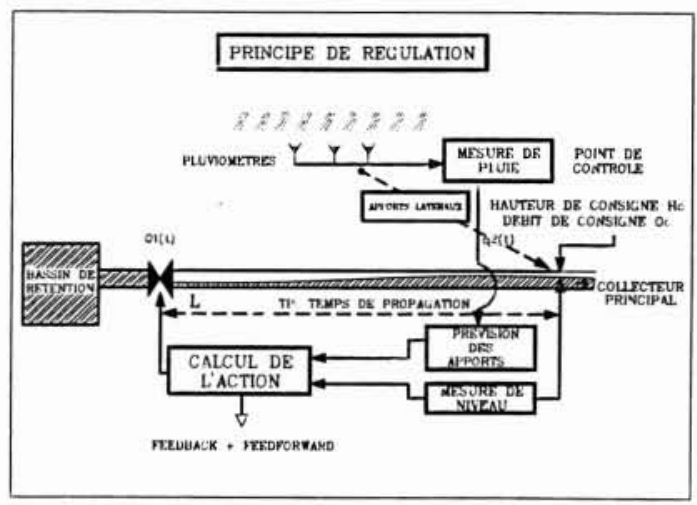

4.
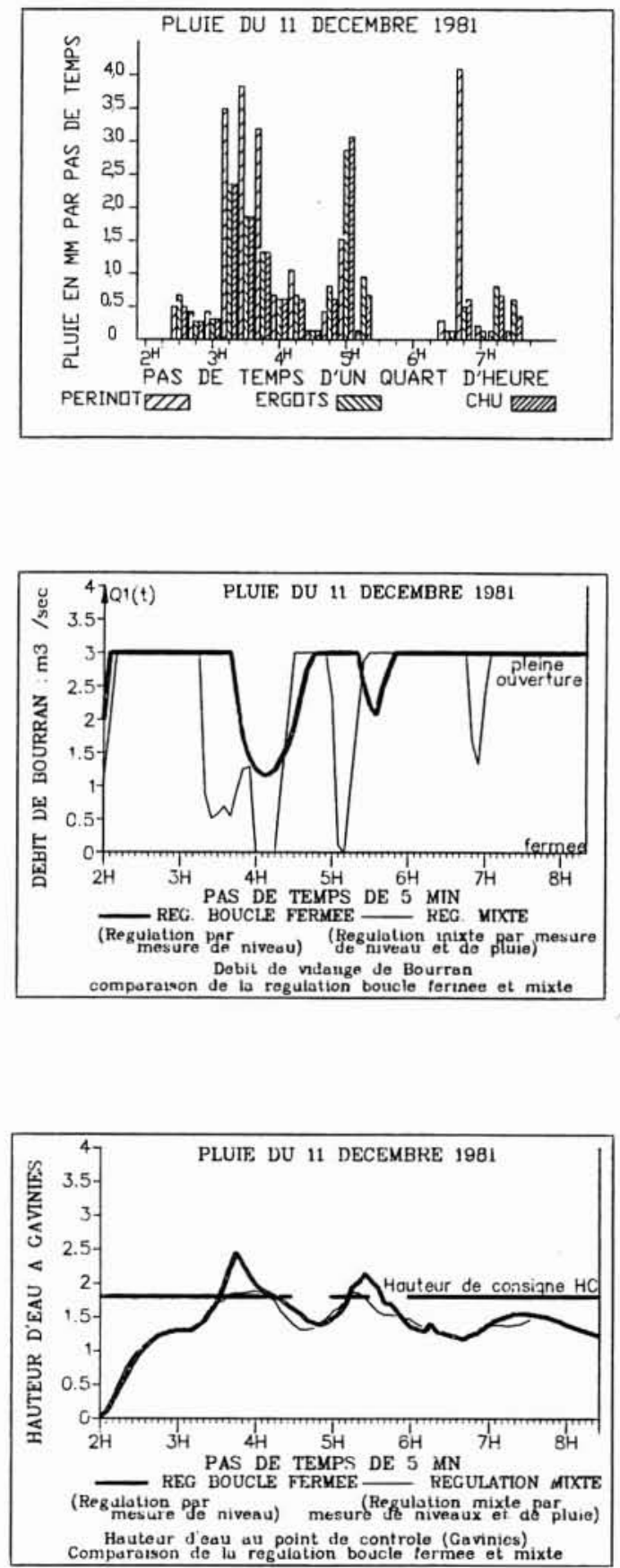

5. 


\section{Conclusions}

La Gestion Technique Centralisée est un moyen efficace et important de la gestion des réseaux d'assainissement, car elle permet de satisfaire les premiers besoins que sont la télésurveillance des équipements, l'acquisition et stockage des paramètres de fonctionnement et des paramètres hydrologiques: limnimétrie et pluviométrie. Ce dernier paramètre occupe d'ailleurs une place particulière car il détermine le déclenchement de phases critiques: mise en alerte, fin alerte...

Les moyens informatiques sont maintenant maîtrisés et deviennent accessibles aux collectivités de taille moyenne. On notera cependant qu'en matière de pluviométrie (prévisions...) des améliorations sont encore attendues des Recherches en cours.

Par ailleurs de nouveaux outils encore au stade expérimental ou en phase d'étude permettrons de satisfaire les autres besoins dont la gestion dynamique des écoulements ; à Bordeaux ceci concerne surtout la vidange des bassins de rétention. 